\title{
Preparation and third-order nonlinear optical property of poly(urethane-imide) containing dispersed red chromophore
}

\author{
F. X. Qiu ${ }^{1,2 *}$ D. Y. Yang 1 , P. P. Li, X. Wang 1 \\ ${ }^{1}$ School of Chemistry and Chemical Engineering, Jiangsu University, Zhenjiang, 212013, China \\ 2Jiangsu Provincial Key Laboratory of Modern Agricultural Equipment and Technology, Zhenjiang 212013, China
}

Received 10 August 2008; accepted in revised form 13 october 2008

\begin{abstract}
A novel poly(urethane-imide) (PUI) containing dispersed red chromophore was synthesized. The PUI was characterized by FT-IR, UV-Vis, DSC and TGA. The results of DSC and TGA indicated that the PUI exhibited high thermal stability up to its glass-transition temperature $\left(T_{g}\right)$ of $196^{\circ} \mathrm{C}$ and $5 \%$ heat weight loss temperature of $229^{\circ} \mathrm{C}$. According to $\mathrm{UV}-\mathrm{V}$ is spectrum and working curve, the maximum molar absorption coefficient and absorption wavelength were measured. They were used to calculate the third-order nonlinear optical coefficient $\chi^{(3)}$. At the same time, the chromophore density of PUI, nonlinear refractive index coefficient and molecular hyperpolarizability of PUI were obtained. The fluorescence spectra of PUI and model compound DR-19 were determined at excitation wavelength $300 \mathrm{~nm}$. The electron donor and acceptor in polymer formed the exciplex through the transfer of the electric charges. The results show that the poly(urethane-imide) is a promising candidate for application in optical devices.
\end{abstract}

Keywords: polymer synthesis, third-order nonlinear optics, molecular hyperpolarizability, nonlinear refractive index coefficient

\section{Introduction}

It is well known that nonlinear optical (NLO) materials can be used for a number of photonic applications, for example, optical signal processing, optical communication, optical computing, electrooptic modulation, optical limiting effect, etc. Polymer is attractive medium for application in optical electronic devices [1-3]. Recently, there has been growing interest in the third-order nonlinearity of azo-dye doped polymer materials for their large $\chi^{(3)}$ value, which are interesting for application in optical-limiting and optical switching application. Photoisomerization of azo-molecules makes it easy to modify linear and nonlinear polarizabilities of molecular as well as optical nonlinear refraction. The optical properties of azo-polymer can be controlled optically, which aroused considerably the interest of people $[4,5]$.
Polyurethane (PU) is a versatile polymer and can be easily prepared by a simple polyaddition reaction of polyol, isocyanate and a chain extender. Unfortunately, the conventional PU is known to exhibit poor thermal stability which limits its applications. Research focused on improving the thermal stability of PU has attempted to achieve this goal in various ways. The most accepted approach for the improvement of thermal stability of PU is a chemical modification in the structure by introducing thermally stable heterocyclic polymers like polyimides [6-8]. Polyimides (PI) are the most important members of heterocyclic polymers with remarkable heat resistance and excellent mechanical, electrical, chemical and durability properties. So the incorporation of polyimide or oligoimide unit into PU has been attempted, resulting in an imide-modified PU (i.e. poly(urethane-imide), 
PUI), which is a type of organic polymer characterized by its outstanding thermal stability, good solubility and film formation ability.

In this paper, the isocyanate groups $(-\mathrm{NCO})$ terminated polyurethane containing a diazo non-linear optical chromophore in the side-chain was synthesized based on toluene diisocyanate (TDI) with two active isocyanate groups and dispersed red 19 (DR19) with two isolated hydroxyl groups, and then the polyurethane terminated with -NCO groups was used to synthesize the poly (urethane-imide) (PUI) with 4,4'-(Hexafluoroisopropylidene) diphthalic Anhydride (6FDA). The structure, the thermal property, third-order nonlinear coefficient and nonlinear refractive index coefficient and molecular hyperpolarizability of the PUI were characterized and obtained. The experimental results showed that the PUI obtained possessed excellent heat-resistance and high third-order nonlinear property.

\section{Experimental}

\section{1. Materials}

Dispersed red 19 was obtained from the ACROS ORGANICS. The 4,4'-(Hexafluoro-isopropylidene) diphthalic Anhydride (6FDA) was obtained from TCI Co. and used without further purification. N,N-dimethylformamide (DMF, A. R.) was provided by Shanghai Gaoqiao petrochemical company. Toluene-2,4-diisocyanate (TDI, industry product) was supplied by Huls Co. and was purified before use.

\subsection{Characterization}

FT-IR spectra of the prepared thin films were obtained on a $\mathrm{KBr}$ pellet using Nicolet AVATAR 360 spectrometer. Thermogravimetric analysis (TGA) and differential scanning calorimetry (DSC) were performed on NETZSCH STA449C. The programmed heating range was from room temperature to $700^{\circ} \mathrm{C}$, at a heating rate of $10^{\circ} \mathrm{C} / \mathrm{min}$ under nitrogen atmosphere. The measurement was taken using 6-10 mg samples. The UV-Vis spectrum of the PUI solution was measured by Shimadzu UV240 spectrophotometer. The photoluminescence measurement was performed on a VARIAN Cary Eclipse spectrofluorophotometer.

\subsection{Preparation of PUI}

The Toluene-2,4-diisocyanate (TDI) $1.25 \mathrm{~g}$ (7.2 mmol) and $15 \mathrm{ml}$ DMF were added into a $250 \mathrm{ml}$ flask equipped with mechanical stirrer, nitrogen inlet, condenser and thermometer to react at room temperature, and then the DR-19 $1.12 \mathrm{~g}$ (3.4 mmol) dissolved in $15 \mathrm{ml}$ DMF was dropped slowly into the system. The reaction was carried out at 40,75 and $90^{\circ} \mathrm{C}$ for $2 \mathrm{~h}$, respectively. After cooling to the room temperature, the 4,4'-(Hexafluoroisopropylidene) diphthalic Anhydride (6FDA) $1.51 \mathrm{~g}(3.4 \mathrm{mmol})$ was dissolved in $30 \mathrm{ml}$ DMAC and dropped into the system and reacted with the -NCO groups at 40 and $90^{\circ} \mathrm{C}$ for $2 \mathrm{~h}$, respectively. The product was deposited in the solution of methanol and water $(\mathrm{V}: \mathrm{V}=1: 1)$. The red poly(urethane-imide) (PUI) was obtained after filtration and

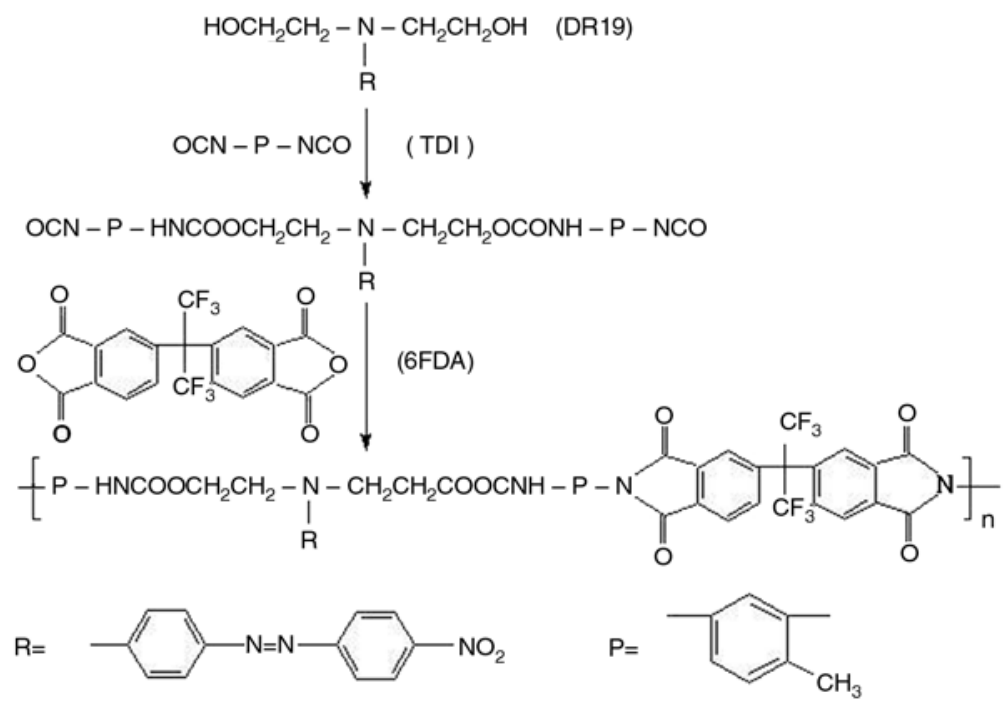

Figure 1. Synthesis of poly (urethane-imide) (PUI) 
drying in vacuum at $60^{\circ} \mathrm{C}$. The synthetic route for PUI is shown in Figure 1.

\section{Results and discussion}

\subsection{Structure characterization and thermal property of PUI}

The UV-Vis spectra of the prepared poly(urethaneimide) material and DR-19 were shown in Figure 2. The content of PUI or DR-19 was $3.0 \cdot 10^{-5} \mathrm{~mol} \cdot \mathrm{l}^{-1}$. From Figure 2, the absorption peak of DR-19 in DMF was $500 \mathrm{~nm}$, while the absorption peak of PUI in DMF was $454 \mathrm{~nm}$. The result indicated that the absorption peak had blue shift after polymerization. The two hydroxyl groups in DR-19 formed urethane bond after polycondensation. The electron cloud in azobenzene groups of DR-19 shifted to the ester bond because of the electron acceptor character of $\mathrm{C}=\mathrm{O}$, which decreased the electron cloud density in azobenzene groups, so absorption band of azobenzene groups would shift to the UV region with the high energy demand of the electron transfer. Because the absorption of PUI was from the azobenzene groups in its side-chain, the UV-Vis data verified the graft reaction of azobenzene monomer with polymer.

Figure 3 illustrates the FT-IR spectrum of the prepared poly(urethane-imide). It shows that the band at $3410 \mathrm{~cm}^{-1}$ is fairly broad and corresponds to the stretching vibration of free and hydrogen-bonded NH groups. Meanwhile, the characteristic absorption bands are observed at 1784,1719 and $1501 \mathrm{~cm}^{-1}$ corresponding to the stretch vibration of $\mathrm{C}-\mathrm{N}$ bond, symmetry coupling vibration of the carbonyl in imide ring, anti-symmetric coupling vibration of

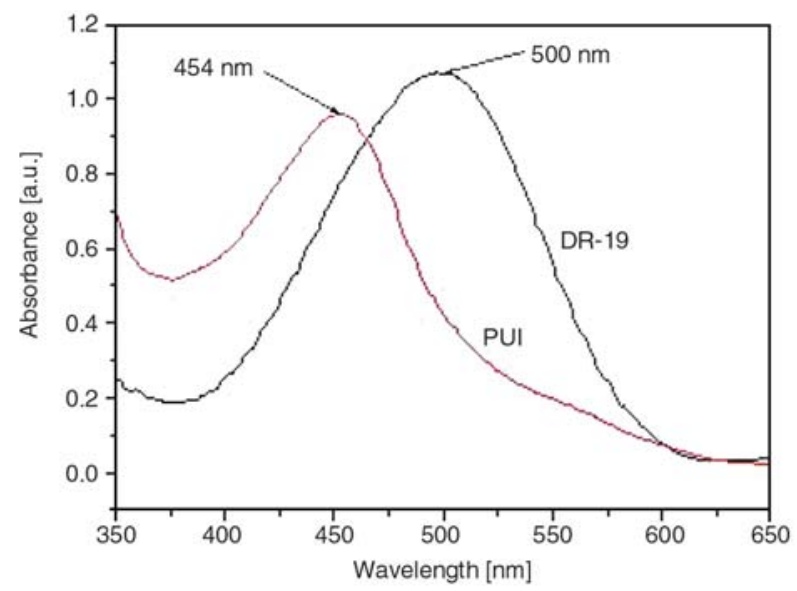

Figure 2. UV-Vis spectra of PUI and DR-19

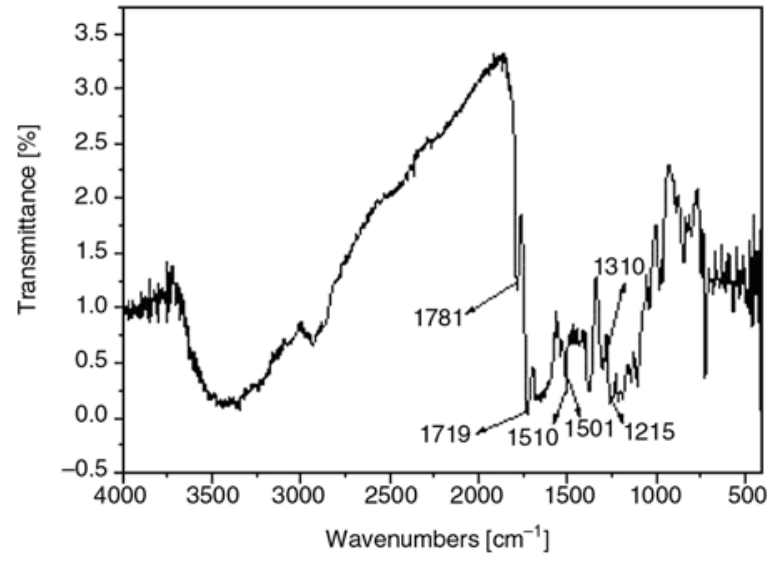

Figure 3. FT-IR spectrum of PUI

the carbonyl in imide ring and the anti-symmetric stretch vibration of $-\mathrm{N}=\mathrm{N}-$ bond, respectively [9]. And the absorption bands at 1510 and $1310 \mathrm{~cm}^{-1}$ are assigned to the symmetric and anti-symmetric stretch vibration of nitro-group in the side-chain of chromophore. $1245 \mathrm{~cm}^{-1}$ is attributed to the ether $(\mathrm{C}-\mathrm{O}-\mathrm{C})$ band of DR-19. These data verified that the imide groups had been introduced to PU backbone. Moreover, the absence of the absorption band at $2270 \mathrm{~cm}^{-1}$ corresponding to the characteristic absorption of isocyanate group indicated that all the monomers were consumed.

To examine thermal activity and thermal decomposition characteristics of material, DSC and TGA experiments were carried out on NETZSCH STA449C with the heating rate $10^{\circ} \mathrm{C} / \mathrm{min}$ under nitrogen, as shown in Figure 4. It can be recognized that the glass transition temperature for PUI was at $196^{\circ} \mathrm{C}$, which is much higher than the corresponding side-chain nonlinear optical (NLO) polyurethanes ( $T_{g}$ of PU based on DR-19 and TDI was at about $137^{\circ} \mathrm{C}$ ). The $5 \%$ heat weight loss tempera-

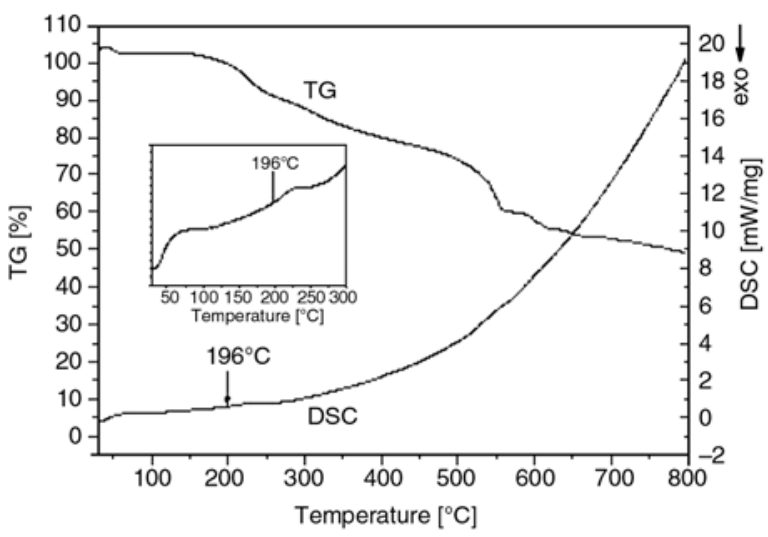

Figure 4. DSC and TGA curves of PUI 
ture of PUI was at $229^{\circ} \mathrm{C}$. When the temperature increased to $780^{\circ} \mathrm{C}$, the mass fraction of PUI was still as high as $50 \%$, indicating the obtained polymer PUI possessed excellent thermal stability. The reason for this was due to the strong hydrogen bonds of the macromolecular chains between polyurethane and polyimide, which formed the physical cross-linkage network among PUI macromolecular chains to alleviate the thermal decomposition of PUI and enhance the thermal stability of this polymer.

\subsection{Determination of third-order nonlinear optical property and molecular hyperpolarizability}

Theory of the nonlinear optical pointed out that the materials with the central non-symmetric structures possessed the second order nonlinear polarization coefficient, even if the arrangement of the molecular orientation was totally random and the molecule was isotropy, the $\chi^{(3)}$ of the material was not equal to zero. The polymer with delocalized $\pi$ electron has high third-order nonlinear optical coefficient and fast response speed, so it was possible to be used in the integrated optic, logic optical path and optical computer. Meisser et al. indicated that there was direct relationships among the nonlinear optical coefficient $\chi^{(3)}(-3 \omega, \omega,-\omega, \omega)$, maximum molar absorption coefficient $\varepsilon_{\max }$ and absorption wavelength $\lambda_{\max }$ of the conjugated polymer [10]. The third-order nonlinear optical coefficient is defined by the Equation (1):

$\chi^{(3)}=\frac{\varepsilon_{\max }}{\lambda_{\max }^{6}}$

The maximum absorption wavelength $\left(\lambda_{\max }\right)$ of PUI was $454 \mathrm{~nm}$ in Figure 2. Different contents of PUI in DMF were prepared and the absorbance was determined at $454 \mathrm{~nm}$ by Shimadzu UV-240 spectrophotometer, respectively. The working curve was obtained and shown in Figure 5. From Figure 5, the linear regression equation was $y=$ $32000 x+0.00493$ ( $y$ was the absorbance, $x$ was concentration of PUI) and the maximum molar absorbance coefficient $\left(\varepsilon_{\max }\right)$ was $32000 \mathrm{~L} \cdot \mathrm{mol}^{-1} \cdot \mathrm{cm}^{-1}$. The correlation coefficient (r) was 0.998 and standard deviation (SD) was $0.664 \%$.

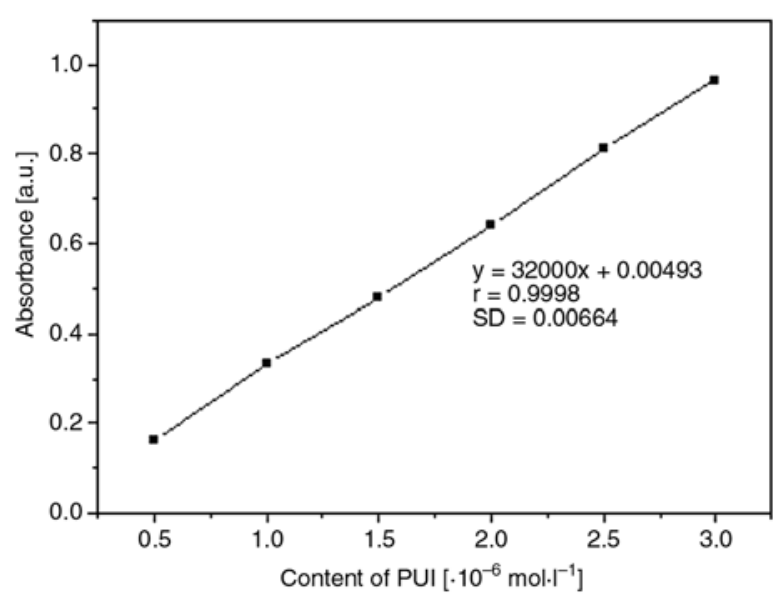

Figure 5. Working curve of PUI

According to the Equation (1), the $\chi^{(3)}$ of PUI was calculated $3.60 \cdot 10^{-12}$ esu. From the data, we could find that the PUI product obtained in this article had a satisfaction third-order nonlinear optical coefficient. Because the diazo bonds connecting with phenyl possessed large $\pi$ electron conjugated structure, it would increase the conjugated degree of the PUI backbone chain and enhance its third-order nonlinear optical coefficient when introduced to the backbone of the polymer as side-chains. In addition, the push-pull structure in PUI macromolecular increased the conjugated length of the diazo bonds in side-chain increased the electron shift of the whole conjugated system and the third-order nonlinear optical coefficient of PUI polymer. Comparing with the theoretical calculation value of $3.10 \cdot 10^{16} \mathrm{molecule} / \mathrm{ml}$, the chromophore density of the prepared PUI measured by the method of working curves was $2.26 \cdot 10^{16}$ molecule $/ \mathrm{ml}$, which was close to the former, indicating that the polymerization was according to the stoichiometric ratio.

Nonlinear refractive index $\left(n_{1}\right)$ coefficient was obtained from third-order nonlinear optical coefficient and described by Equation (2) [11]:

$n_{1}=\frac{12 \pi \chi^{(3)}}{n_{\text {sample }}^{2}}$

where $n_{\text {sample }}$ is refractive index of PUI $(n=1.415)$. According to this formula (2), nonlinear refractive index coefficient of PUI is $6.78 \cdot 10^{-11}$ esu.

Molecular hyperpolarizability $(\gamma)$ of sample can be calculated and expressed by Equation (3): 


$$
\gamma=\frac{\chi^{(3)}}{T^{4} N_{0}}
$$

where $N_{0}$ is the number of molecule per milliliter $\left(2.26 \cdot 10^{16} \mathrm{molecule} / \mathrm{ml}\right) . T$ is local field revision factor, it can be expressed by Equation (4):

$$
T=\frac{n_{\text {sample }}^{2}+2}{3}
$$

According to the formula (4) and (3), $T$ and $\gamma$ were 1.334075 and $5.03 \cdot 10^{-29}$ esu, respectively. The results indicated that PUI had high third-order nonlinear polarizability.

\subsection{Fluorescence characterization of PUI}

The fluorescence characters of PUI and model compound DR-19 were determined at excitation wavelength of $300 \mathrm{~nm}$. The Fluorescence spectra were obtained in Figure 6.

It showed that both in the spectrum of PUI and DR19 there appeared fluorescence peaks and their fluorescence effects were similar, which indicated that the fluorescence effect of PUI was generated from the azobenzene groups in its macromolecular chains. But at the same concentration, the fluorescence intensity of PUI was lower than that of DR19, which may be a result of the special structure of PUI. In the molecular chain of PUI, there were different length of non-conjugated chains (which came from the $-\mathrm{O}\left(\mathrm{CH}_{2}\right)_{2} \mathrm{~N}-$ in $\mathrm{DR}-19$ and $-\mathrm{HNCCH}_{2} \mathrm{CNH}-$ in TDI) and definite length of conjugated chains, which forming the un-luminiferous and luminiferous chains of polymer PUI. At the same time, in PUI there existed both small size

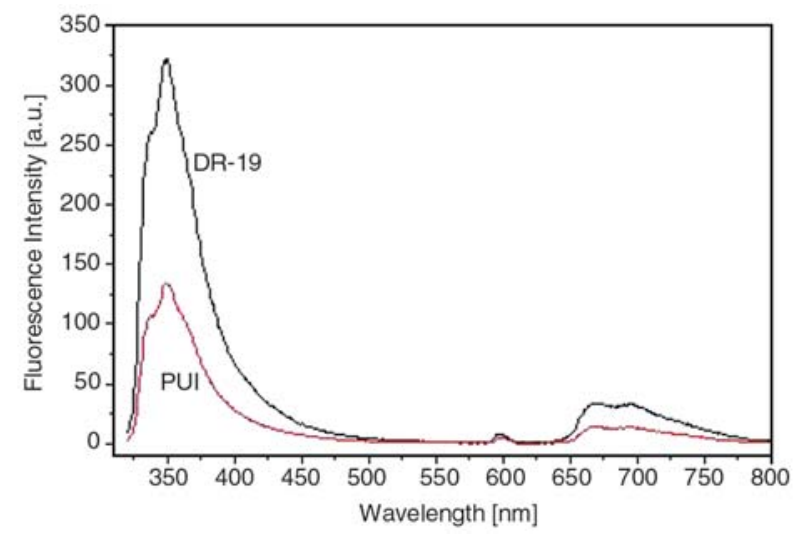

Figure 6. Fluorescence spectra of DR-19 and PUI and strong stiffness and carbonyl with electron donor groups and electron acceptor of 6FDA, which formed the intramolecular or intermolecular exciplex through the transfer of the electric charges at condition of lighting. So the fluorescence intensity of PUI decreased with the self-quenching of the macromolecules in ground state and excited state when transferring among the molecules $[12,13]$.

\section{Conclusions}

We synthesized a novel poly(urethane-imide) (PUI) containing a diazo nonlinear optical chromophore (dispersed red 19) in the side-chain. The determination results of the glass-transition temperature $\left(T_{g}\right)$ and $5 \%$ heat weight loss temperature indicated that the material possessed excellent thermal stability. Measurement result of the chromophores density of PUI showed that the polymerization was according to the stoichiometric ratio. The PUI has high third-order nonlinear coefficient and polarizability. Moreover, its fluorescence effect was result from the azobenzene groups in its side-chains. The electron donor and acceptor in polymer formed the exciplex through the transfer of the electric charges at condition of lighting. These results show that PUI polymer is promising materials for nonlinear optical applications.

\section{Acknowledgements}

This project was supported by the Natural Science of Jiangsu Province (BK2008247), China Postdoctoral Science Foundation (20070420973), the Jiangsu Planned Projects for Postdoctoral Research Funds (0602037B), Jiangsu Provincial Key Laboratory for Science and Technology of Photon Manufacturing and the Natural Science of Jiangsu Education (08KJB150004).

\section{References}

[1] Qiu F. X., Cao Y. L., Xu H. L., Jiang Y., Zhou Y. M., Liu J. Z.: Synthesis and properties of polymer containing azo-dye chromophores for nonlinear optical applications. Dyes and Pigments, 75, 454-459 (2007).

[2] Qiu F. X., Da Z. L., Yang D. Y., Cao G. R., Li P. P.: The synthesis and electro-optic properties of polyimide/silica hybrids containing the benzothiazole chromophore. Dyes and Pigments, 77, 564-569 (2008). 
[3] Qiu F. X., Li P. P., Yang D. Y.: Synthesis, characterizations and electro-optical properties of nonlinear optical polyimide/silica hybrid. Express Polymer Letters, 1, 150-156 (2007).

[4] Fuh A. Y-G., Lin H-C., Mo T-S., Chen C-H.: Nonlinear optical property of azo-dye doped liquid crystals determined by biphotonic Z-scan technique. Optics Express, 13, 10634-10641 (2005).

[5] Lee M-R., Wang J-R., Lee C-R., Fuh A. Y-G.: Optically switchable biphotonic photorefractive effect in dye-doped liquid crystal films. Applied Physics Letters, 85, 5822-5824 (2004).

[6] Gnanarajan T. P., Nasar A. S., Iyer N. P., Radhakrishnan G.: Synthesis of poly(urethane-imide) using aromatic secondary amine-blocked polyurethane prepolymer. Journal of Polymer Science Part A: Polymer Chemistry, 38, 4032-4037 (2000).

[7] Lin M-F., Shu Y-C., Tsen W-C., Chuang F-S.: Synthesis of polyurethane-imide (PU-imide) copolymers with different dianhydrides and their properties. Polymer International, 48, 433-445 (1999).

[8] Chen J. N., Zhang J. F., Zhu T. Y., Hua Z. C., Chen Q. M., Yu X. H.: Blends of thermoplastic polyurethane and polyether-polyimide: Preparation and properties. Polymer, 42, 1493-1450 (2001).
[9] Qiu F. X., Xu H. L., Cao Y. L., Jiang Y., Zhou Y. M., Liu J. Z., Zhang X. P.: Nonlinear optical materials: Synthesis, characterizations, thermal stability and electro-optical properties. Materials Characterization, 58, 275-283 (2007).

[10] Messier J., Kajzar F., Prasad P.: Organic molecules for nonlinear optics and photonics. Kluwer Academic Publishers, Dordrecht (1991).

[11] Jenekhe S. A., Lo S. K., Flom S. R.: Third-order nonlinear optical properties of a soluble conjugated polythiophene derivative. Applied Physics Letters, 54, 2524-2526 (1989).

[12] Hong Z. Y., Zhao X. J., Ma D. G., Wang D. K., Jing X. B., Wang F. S.: Influence of fluorescence intensity for polymeric structure and chain distance. Chinese Science Bulletin, 43, 588-592 (1998).

[13] Zhang X. L., Yuan J. F., Qu J. P., Gao Q. Y.: Synthesis of (4-N,N-dimethylamine cinnamoylat ethyl) acrylate and its water soluble copolymers and their photochemical property. Photographic Science and Photochemistry, 23, 41-47 (2005). 